\title{
Criticality of environmental information obtainable by dynamically controlled quantum probes
}

\author{
Analia Zwick, Gonzalo A. Álvarez and Gershon Kurizki ${ }^{1}$ \\ ${ }^{1}$ Weizmann Institute of Science, Rehovot 76100, Israel
}

\begin{abstract}
A universal approach to decoherence control combined with quantum estimation theory reveals a critical behavior, akin to a phase transition, of the information obtainable by a qubit probe concerning the memory time of environmental fluctuations. This criticality emerges only when the probe is subject to dynamical control. It gives rise to a sharp transition between two dynamical phases characterized by either a short or long memory time compared to the probing time. This phase-transition of the environmental information is a fundamental feature that facilitates the attainment of the highest estimation precision of the environment memory-time and the characterization of probe dynamics.
\end{abstract}

PACS numbers: 03.65.Ta, 03.65.Yz, 03.67.-a, 64.70.qj

Keywords:

A simple quantum-probe, such as a qubit, is capable of extracting information on the environment dynamics and its space-time fluctuations through the spectrum of the dephasing noise the probe is subject to [1-14]. This information is the subject of an emerging field of research dubbed environmental quantum-noise spectroscopy [5, 6]. Its most straightforward implementation is by monitoring the freeinduction decay of an initially-prepared qubit-probe coherence and inferring the dephasing characteristics from this decay $[15,16]$. A more promising option is to exert a control (driving) field, whether pulsed or continuouswave $(\mathrm{CW})$, on the qubit-probe and study its dephasing as a function of the control-field characteristics [4-6]. Pulsed control of qubit dephasing is commonly described by dynamical decoupling [17-21]. However, for the purpose of environment-noise spectroscopy it is useful to resort to the universal formula for the rate of decoherence under dynamical control [22-25], which is at the heart of the unified theory of dynamically-controlled open quantum systems [26-28]. This universal formula allows the design of control fields or pulse sequences that through the choice of a spectral filter function are optimally tailored to the specific environment-noise spectrum and the task at hand [25, 29]: decoherence control [22, 23, 26, 27, 30-34], state-transfer $[35,36]$ or storage [36-38] in a fluctuating environment. Here, the filter function will be optimally adapted to the task of probing the environment-noise spectrum by a qubit [39].

Among environment-noise parameters whose estimation is of practical interest in physics, chemistry and biology, the memory or correlation time is particularly helpful [48, 39-51]. On a fundamental level, memory effects of the environment are associated with the concept of nonMarkovianity, whose definition is an outstanding issue [52, 53].

For the purpose of characterizing the memory effects of an environment that interacts with a qubit probe, we here put forward an approach based on the aforementioned universal formula for decoherence control [22-29] combined with quantum estimation theory [39, 54-57]. We show that the information (estimation-precision) concerning the environment-noise fluctuation-spectrum obtained by this approach may exhibit critical behavior as a function of the memory-time parameter. This critical behavior, akin to a phase transition, is only revealed under dynamical control: it defines a sharp boundary between the short- and longtime regimes of the probe decoherence corresponding to long- and short-memory of the environment respectively. By contrast, free-induction decay of the probe coherence undergoes the usual smooth transition between the tworegimes, thus conforming to the gradual change from nonMarkovianity to Markovianity that has been previously analyzed $[52,53]$. The criticality or phase-transition of the environmental information revealed here is a fundamental feature that characterizes dynamical behavior with practical implications on the attainment of the highest estimation precision of the environment memory-time (see Discussion).

Controlled qubit-probe as a sensor of the environmental fluctuations. - We consider a dynamically-controlled qubit-probe experiencing pure dephasing in the weakcoupling probe-environment regime (Fig. 1a), which is characterized by the attenuation (decay) factor $\mathcal{J}\left(\vec{x}_{B}, t\right)$ of the qubit coherence [39]

$$
\left\langle\sigma_{x}(t)\right\rangle=\sigma_{x}(0) e^{-\mathcal{J}\left(\vec{x}_{B}, t\right)},
$$

where $\vec{x}_{B}$ are a set of parameters that describe the environment and the attenuation factor obeys the universal formula [22-29]

$$
\mathcal{J}\left(\vec{x}_{B}, t\right)=\int_{-\infty}^{\infty} d \omega F_{t}(\omega) G\left(\vec{x}_{B}, \omega\right) .
$$

Here $G\left(\vec{x}_{B}, \omega\right)$ is the coupling spectrum (spectral density) of the environment noise (the Fourier transform of its autocorrelation function). Explicitly, $\vec{x}_{B}=\left[g, \tau_{c}, \beta\right]$, with $\tau_{c}$ as the correlation or memory time of the environment noise, i.e. the inverse width of its spectral density, $g$ as the effective probe-environment coupling-strength, and $\beta$ as a power law exponent that defines the type of stochastic (noise) process. The filter function $F_{t}(\omega)$ explicitly depends upon the dynamical control of the probe during time 


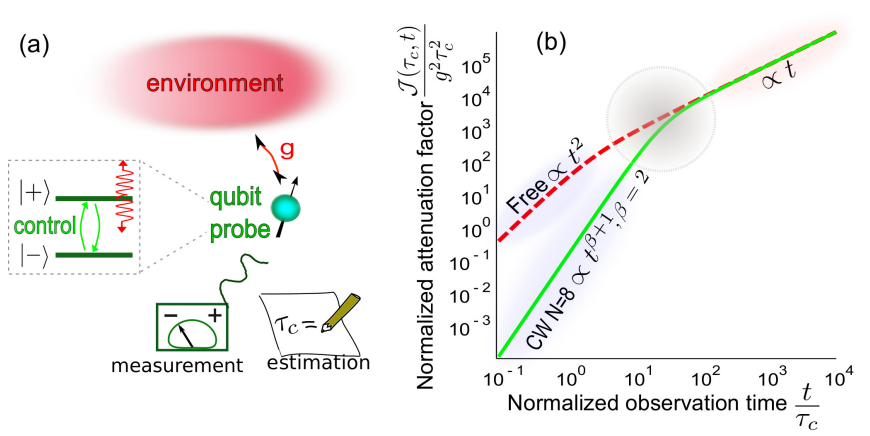

Figure 1: (a) Estimation of the environmental noise fluctuations by a qubit that probes the environment. A dynamically controlled qubit-probe undergoes pure-dephasing by the environment due to the interaction $H_{S B}=g \sigma_{z} B$, where $\sigma_{z}$ is the Pauli operator for the probe and $B$ is the environment (bath) operator. The dephasing is revealed by the attenuation (decay) factor $\mathcal{J}$ that characterizes the optimal qubit-observable $\left\langle\sigma_{x}\left(\vec{x}_{B}, t\right)\right\rangle=e^{-\mathcal{J}\left(\vec{x}_{B}, t\right)}$, $\vec{x}_{B}$ being the set (unknown) noise parameters, for an (optimal) initial state - the symmetric superposition of the spin-up/-down states in the basis $\sigma_{z}, \frac{1}{\sqrt{2}}(|\uparrow\rangle+|\downarrow\rangle)=|+\rangle[39,57,60]$. Here we focus in estimating $x_{B}=\tau_{c}$. (b) Time dependence of the normalized attenuation factor $\mathcal{J}$ of the qubit-state probing an OrnsteinUhlenbeck process $(\beta=2)$ for free evolution (dashed) compared to its counterpart under dynamical control (solid). The latter time dependence exhibits a smooth transition (marked by a circle) between two well-defined dynamical phases (regimes) depending on the ratio $\frac{t}{\tau_{c}}$.

$t$. The information about the unknown environment parameters $\vec{x}_{B}$ is encoded by the probabilities $p$ of finding the qubit-probe in the $|+\rangle$ (symmetric) or $|-\rangle$ (antisymmetric) superposition of the qubit energy states when measuring $\sigma_{x}$. These probabilities obey

$$
p_{ \pm}\left(\vec{x}_{B}, t\right)=\frac{1}{2}\left(1 \pm e^{-\mathcal{J}\left(\vec{x}_{B}, t\right)}\right) .
$$

As a model to describe the memory time scales of the environment, we consider a generalized Ornstein-Uhlenbeck spectral density [39]

$$
G_{\beta}\left(\left[g, \tau_{c}, \beta\right], \omega\right)=g^{2} \frac{\mathcal{A}_{\beta} \tau_{c}}{1+\omega^{\beta} \tau_{c}^{\beta}},
$$

where $\mathcal{A}_{\beta}$ is a normalization factor depending on the power-law $\beta \geq 2$. In fact, this model is the building block of universal lineshapes: it may characterize the memory time of arbitrary bosonic environments, if one assumes that a chosen harmonic-oscillator mode constitutes an interface between the qubit-probe and the modes of the environment [58]. The combined spectrum of any environment plus the interface mode is then reshaped, or "filtered", according to the chosen oscillator-mode frequency and its coupling strength with the probe, invariably resulting in a skewedLorentzian lineshape $[58,59]$.

The power law regime $\alpha \omega^{-\beta}$ of the spectral density, obtained for $\omega \tau_{c} \gg 1$, is the spectral range with the strongest

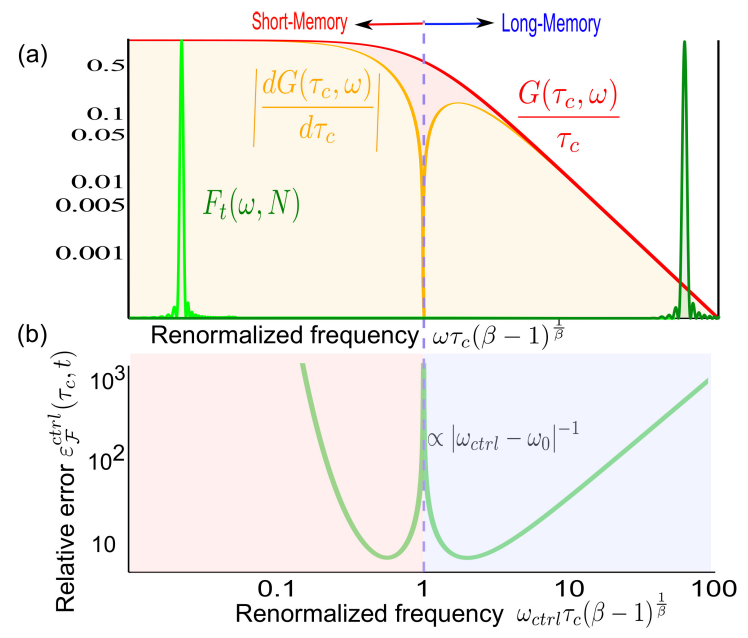

Figure 2: Criticality of the probe-extracted information on the environmental correlation (memory) time $\tau_{c}$. (a) Spectral density for the Ornstein-Uhlenbeck process (Lorentzian spectrum, $\beta=$ 2: red solid line). The derivative of the spectrum $\left|\frac{d G}{d \tau_{c}}\right|$ exhibits a critical behavior $\left|\frac{d G}{d \tau_{c}}\right| \propto\left|\omega-\omega_{0}\right|$ at $\omega_{0}=\tau_{c}^{-1}(\beta-1)^{-\frac{1}{\beta}}$. The two dynamical regimes occur when the narrow filters probe frequency components of $G(\omega)$ on both sides of the critical point. Two typical continuous wave (CW-control) filter functions $F_{t}(\omega)$ (green, in linear scale) scan the spectrum on both sides of the transition $(N=20)$. (b) The attainable relative error $\varepsilon_{\mathcal{F}}^{c t r l}\left(\tau_{c}, t=\right.$ $\left.\frac{\pi N}{\omega_{c t r l}}\right)$ on $\tau_{c}$ by the qubit-probe under $\mathrm{CW}$ control $\left(\sqrt{2 N} g \tau_{c}=1\right.$, $\beta=2$ and $\left.\omega_{c t r l}=\frac{\pi N}{t}\right)$. The divergence $\propto\left|\omega_{c t r l}-\omega_{0}\right|^{-1}$ at the critical point gives evidence of the critical behavior.

dependence on the frequency $\omega$, describing the short time behavior of the probe-qubit dephasing. We define this limit as the Long Memory (LM) regime. In the opposite limit $\omega \tau_{c} \ll 1$, associated with long times, the spectral density becomes independent of the frequency, and the probe coherence attenuation factor $\mathcal{J}\left(\vec{x}_{B}, t\right)$ is then given by the Fermi Golden Rule. We dub this limit the Short Memory (SM) regime.

Identifying the dynamical regimes' criticality by dynamically controlled probes. - Under free induction decay, the LM and SM regimes are attained at times $t \ll \tau_{c}$ and $t \gg \tau_{c}$, respectively. The respective decay (attenuation) factors are $\mathcal{J}_{\text {free }}^{L M} \propto g^{2} t^{2}$ (independent of $\tau_{c}$ ) and $\mathcal{J}_{\text {free }}^{S M} \propto g^{2} \tau_{c} t$, by considering $F_{t}^{\text {free }}(\omega)=\frac{t^{2} \operatorname{sinc}^{2}\left(\frac{\omega t}{2}\right)}{2}$ [39]. The transition from the LM to the SM regime is smooth (Fig. 1b) as the ratio $\frac{t}{\tau_{c}}$ is varied and does not depend on g. Invariably, $\frac{\partial \mathcal{J}_{\text {free }}}{\partial \tau_{c}}>0$, without sign change.

Consider now the change that may arise in the character of this transition under dynamical control. An example is a decoupling control sequence of $N \gg 1$ equidistant $\pi$-pulses (known as CPMG) [16, 61, 62]. The filter function $F_{t}(\omega)$ [4, 22-29] then converges to a sum of delta functions (narrowband filters) centered at the harmonics of the inverse modulation period, $k \omega_{c t r l}=\frac{\pi k N}{t}$ 
with $k=1,2,3, .$. [5, 34]. Another suitable control is $\mathrm{CW}$ qubit-driving, which has a single frequency component $(k=1)$. Under such controls, the LM and SM regimes are attained for $\omega_{c t r l} \tau_{c} \gg 1$ and $\omega_{c t r l} \tau_{c} \ll 1$, respectively. The corresponding decay factors, $\mathcal{J} \propto F_{t}\left(\omega_{c t r l}\right) G\left(\omega_{c t r l}\right)$ in Eq. (2), are (see Fig. 1b)

$$
\mathcal{J}^{L M} \propto \frac{g^{2} t}{\omega_{c t r l}^{\beta} \tau_{c}^{\beta-1}}, \mathcal{J}^{S M} \propto g^{2} \tau_{c} t
$$

respectively. This reflects the effect of a narrow-band filter $F_{t}\left(\omega_{c t r l}\right)$ that may be used to scan the spectral density $G(\omega)$ [4-6], upon varying the modulating frequency (pulse-rate or Rabi-frequency) $\omega_{c t r l}$ of the control field, all the way from the frequency-independent regime $G \propto \tau_{c}$ for $\omega \tau_{c} \ll 1$ to the power-law regime $G \propto \omega^{-\beta} \tau_{c}^{-(\beta-1)}$ for $\omega \tau_{c} \gg 1$ (Fig. 2a). In the limit of extremely narrow spectral filters, i.e. $N \rightarrow \infty$, with $\omega_{c t r l}=\frac{\pi N}{t}$, we have

$$
\begin{gathered}
\left.\left.\frac{\partial \mathcal{J}}{\partial \tau_{c}}\right|_{\omega_{c t r l} \sim \omega_{0}} \propto \frac{\partial G}{\partial \tau_{c}}\right|_{\omega_{c t r l} \sim \omega_{0}} \propto \omega_{c t r l}-\omega_{0} ; \\
\omega_{0}=\frac{1}{\tau_{c}(\beta-1)^{\frac{1}{\beta}}} .
\end{gathered}
$$

An abrupt change (Fig. 2a) is then revealed in the parametric sensitivity of the attenuation-factor derivative $\frac{\partial \mathcal{J}}{\partial \tau_{c}}$ through its change of sign: $\frac{\partial \mathcal{J}}{\partial \tau_{c}} \propto \frac{-(\beta-1) \mathcal{J}^{L M}}{\tau_{c}}<0$ for LM and $\frac{\partial \mathcal{J}}{\partial \tau_{c}} \propto \frac{\mathcal{J}^{S M}}{\tau_{c}}>0$ for SM, implying that

$$
\left.\frac{\partial \mathcal{J}}{\partial \tau_{c}}\right|_{\omega_{c t r l} \tau_{c}(\beta-1)^{\frac{1}{\beta}} \approx 1}=0
$$

at a value dependent on the control (modulating) frequency $\omega_{\text {ctrl }}$.

The central result of this paper is that Eq. (8) signifies the vanishing of the quantum Fisher information (QFI) [54], which quantifies the attainable amount of information on $\tau_{c}$ that can be extracted from the measured probe-(qubit) state probabilities $p_{ \pm}$obeying Eq. (3). This vanishing becomes apparent upon considering the expression for QFI $[39,60]$

$$
\mathcal{F}_{\mathcal{Q}}\left(\tau_{c}, t\right)=\frac{e^{-2 \mathcal{J}}}{1-e^{-2 \mathcal{J}}}\left(\frac{\partial \mathcal{J}}{\partial \tau_{c}}\right)^{2} .
$$

Hence, at the value $\omega_{c t r l} \tau_{c}(\beta-1)^{\frac{1}{\beta}} \approx 1$ no information can be extracted, $\mathcal{F}_{\mathcal{Q}}{ }^{c t r l}=0$. Since the minimum achievable relative error (per measurement) of the (unbiased) estimation of $\tau_{c}$ is related to the QFI through the Cramer-Rao bound as

$$
\frac{\delta \tau_{c}}{\tau_{c}} \geq \varepsilon_{\mathcal{F}}\left(\tau_{c}, t\right)=\frac{1}{\tau_{c} \sqrt{\mathcal{F}_{\mathcal{Q}}\left(\tau_{c}, t\right)}},
$$

this error diverges as $\omega_{c t r l} \rightarrow \omega_{0}$ (Eq. (7)):

$$
\varepsilon_{\mathcal{F}}^{c t r l}\left(\tau_{c}, t \approx \frac{\pi N}{\omega_{c t r l}}\right) \propto\left|\omega_{c t r l}-\omega_{0}\right|^{-1}
$$

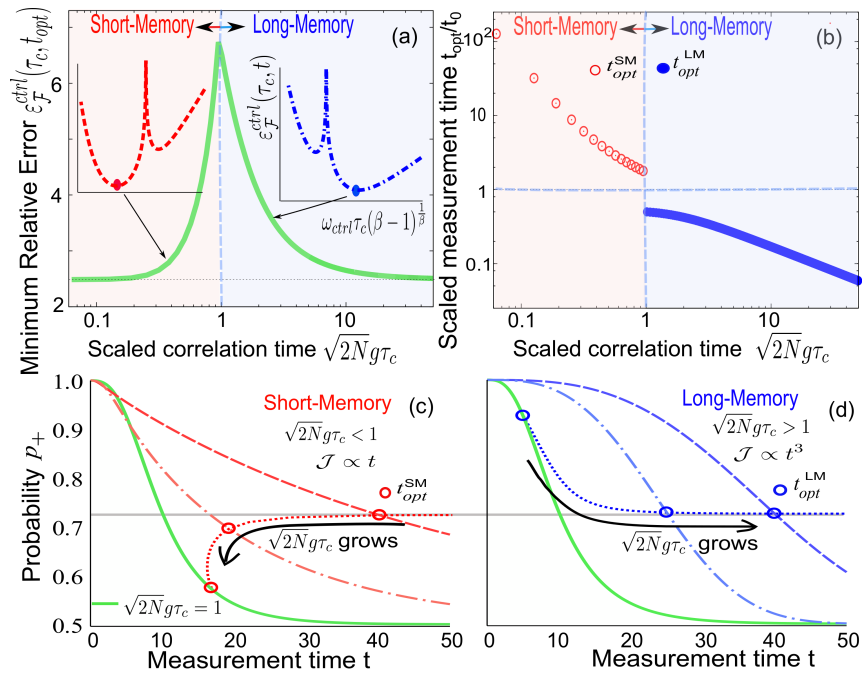

Figure 3: Critical transition of the minimal error in environment memory-time estimation determined by a probe under dynamical control CW in the narrow filter approximation. The noise spectrum is a Lorentzian $(\beta=2)$. (a) The minimum relative error per measurement $\varepsilon_{\mathcal{F}}^{c t r l}\left(\tau_{c}, t_{\text {opt }}\right)$ of the environmental correlation time $\tau_{c}$, as a function of $\sqrt{2 N} g \tau_{c}$, obtained by optimizing the control time $t_{\text {opt }}$. It exhibits a critical behavior at $\sqrt{2 N} g \tau_{c} \approx 1$. Insets: $\varepsilon_{\mathcal{F}}^{c t r l}\left(\tau_{c}, t=\frac{\pi N}{\omega_{c t . r l}}\right)$ for global minima located in the SM (left inset) and LM (right inset) regimes. The critical point emerges when both local minima are equal (as in Fig. 2b). (b) The optimal scaled measurement time $\frac{t_{o p t}}{t_{0}}$ as a function of $\sqrt{2 N} g \tau_{c}$. (c-d) Probability $p_{+}(t)$ as a function of time. For $\sqrt{2 N} g \tau_{c}<1$, the optimal time $t_{\text {opt }}^{S M}$ (red circles) corresponds to a linear attenuation factor $\mathcal{J}^{S M} \propto t$ (panel (c) and Fig. (1)b), while for $\sqrt{2 N} g \tau_{c}>1$, the optimal time $t_{o p t}^{L M}$ (blue solid circles) belongs to a regime where $\mathcal{J}^{L M} \propto t^{\beta+1}$ (panel (d)). At $\sqrt{2 N} g \tau_{c} \approx 1$ (vertical dashed line) the transition between the two regimes is observed. The optimal time jumps between $t_{\text {opt }}^{L M}$ and $t_{\text {opt }}^{S M}$ at the critical point avoiding the time $t_{0}=\pi N \tau_{c}(\beta-1)^{\frac{1}{\beta}}$ (horizontal dashed line) where no-information about $\tau_{c}$ can be extracted from the probe.

The relative error, $\varepsilon_{\mathcal{F}}^{c t r l}$, thus exhibits a sharp transition between the LM and SM dynamical regimes (Fig. 2b), allowing their clear distinction. This critical behavior of the relative error provides a signature of the environmental noise spectral density through the values of $\tau_{c}$ and $\beta$, provided we apply an appropriate dynamical control that generates a sufficiently narrow spectral filter, so as to scan the sign change of $\left.\frac{\partial G}{\partial \tau_{c}}\right|_{\omega \sim \omega_{0}}$ at the critical point (Fig. 2). By contrast, such criticality does not arise under free-induction decay, for which the filter function, $F_{t}^{\text {free }}(\omega)$ is a much broader sinc function centered around $\omega=0$.

Critical behavior of the maximal estimation precision of $\tau_{c}$ - - The critical behavior shown above is also manifest, under the same control on the probe, for the maximized estimation precision, i.e. the smallest possible minimal rela- 


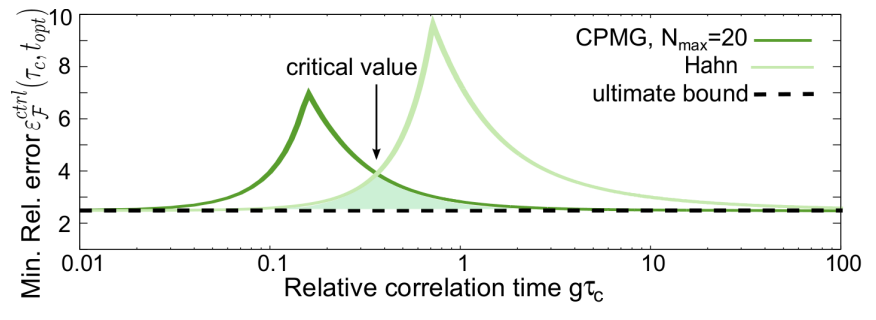

Figure 4: Minimal relative error per measurement in the estimation of $\tau_{c}$ as a function of $g \tau_{c}$ for a Lorentzian environmental spectrum under dynamical control. Practical limitations on the number of pulses of a CPMG sequence, such that $N \leq N_{\max }$, may prevent to attainment of the ultimate bound (dashed line) [39]. Then, a sudden change of the dynamical control strategy as a function of $g \tau_{c}$ may help: For $g \tau_{c}$ lower than the critical value, the highest precision is achieved by the single-pulse Hahn echo $(N=1)$. However, if $g \tau_{c}$ is larger than the critical value CPMG sequence with $N=N_{\max }$ is optimal. This dynamical control strategy under practical limitations reduces the minimal error represented by the shaded area, which is determined by the optimal control on each side of the intersection (critical value) of the Hahn and the CPMG curves.

tive error in the estimation of $\tau_{c}$ in Eq. (10),

$$
\varepsilon_{\mathcal{F}}^{c t r l}\left(\tau_{c}, t_{\text {opt }}\right)=\min _{t} \varepsilon_{\mathcal{F}}^{c t r l}\left(\tau_{c}, t\right) .
$$

The error minimization is the outcome of selecting the optimal time $t_{o p t}$ at which the measurement (cf. Eqs. (1), (2)) is performed on the probe, following its dephasing under the control we have applied.

Figure 3 shows the critical behavior of the maximum precision per measurement, $\varepsilon_{\mathcal{F}}^{c t r l}\left(\tau_{c}, t_{\text {opt }}\right)$ for the Lorentzian spectrum $(\beta=2)$ following $\mathrm{CW}$ control of the qubit-probe as a function of $\sqrt{2 N} g \tau_{c}$. The critical point

$$
\sqrt{2 N} g \tau_{c} \approx 1
$$

separates two regions characterized by different scalinglaws of the minimal relative-error as a function of $\sqrt{2 N} g \tau_{c}$ (Fig. 3a). These scaling-laws are dictated by the different dynamical regimes for the attenuation (decay) factor shown in Figs. 3c,3d.

The optimal probing (measurement) and control time $t_{o p t}$ is also shown to undergo a sudden transition at the critical point (13), as shown in Fig. 3b. This optimal time corresponds to the best tradeoff between a signal amplitude contrast, $\frac{e^{-2 \mathcal{J}}}{1-e^{-2 \mathcal{J}}}$, and the parametric sensitivity of the signal attenuation factor, $\left(\frac{\partial \mathcal{J}}{\partial \tau_{c}}\right)^{2}$. The optimal tradeoff occurs (Fig. 3b) at either a long time, $t_{\text {opt }}^{S M}$ (red circle) corresponding to a linear attenuation factor $\mathcal{J}^{S M} \propto t$ (Fig. $3 \mathrm{c}$ ) or at a short time, $t_{\text {opt }}^{L M}$ (blue circle) corresponding to $\mathcal{J}^{L M} \propto t^{\beta+1}$ (Figs. 3d). These optimal control times in the two regimes are situated on both sides of the critical value (Fig. 3b)

$$
t_{\text {opt }}^{L M}<t_{0}<t_{\text {opt }}^{S M}, \quad t_{0}=\frac{\pi N}{\omega_{0}} .
$$

The criticality of the relative error as a function of $\omega_{c t r l}$ described by Eq. (11), defines two local, unequal minimum values of $\varepsilon_{\mathcal{F}}^{c t r l}$, located on either side of the critical point (Fig. 2b and insets Fig. 3a). What determines the global minima of $\varepsilon_{\mathcal{F}}^{c t r l}$ is the optimal time $t_{\text {opt }}$ obtained from Eq. (12). The critical behavior emerges when this global minimum jumps between the two local minima as a function of the parameter $\sqrt{2 N} g \tau_{c}$ (Fig. 3b). At the critical point, both local minima are equal, as displayed in Fig. 2 b.

Discussion. - We have demonstrated a critical behavior of information (estimation-precision) on the environment fluctuation (noise) spectrum of generalized OrnsteinUhlenbeck process extracted by a probe subject to appropriate dynamical control as a function of the environment memory-time $g \tau_{c}$. This finding applies to any bosonic environment, provided the probe and the environment are suitably interfaced by a chosen oscillator mode [58, 59] (cf. discussion following Eq. (4)).

We have shown that similar critical behavior is manifest for the maximal estimation precision of $\tau_{c}$. At the critical point there is a massive loss of information on $\tau_{c}$. Near this point, the optimal time for measuring and controlling the quantum-probe is either very short, corresponding to little parametric sensitivity, or very long, corresponding to significant decay of the signal.

The critical behavior of the maximal estimation precision of $\tau_{c}$ has paramount practical implications:

(i) Complete dynamical behavior characterization: Rather than mapping out the long- and short-memory probe dynamics regimes by varying the probing time, the critical behavior demonstrated here, allows one to characterize the complete dynamics as consisting of two distinct dynamical phases (regimes) according to the maximal information they yield near the critical point (13).

(ii) Sudden change of the optimal dynamical control sequence: The critical point depends on the control scheme: thus, for CPMG control $[16,61,62] g \tau_{c} \approx \frac{1}{\sqrt{2 N}}$ when probing a Ornstein-Uhlenbeck process (Lorentzian spectra). This fact highlights the importance of optimizing the number of pulses $N$ so as to improve the estimation precision, if $N$ is bounded by $N_{\max }$ due to practical limitations. Under these conditions, the ultimate bound on the estimation precision found in Ref. [39]

$$
\varepsilon_{\mathcal{F}}\left(\tau_{c}, t\right) \geq \varepsilon_{0},
$$

where $\varepsilon_{0}=\frac{\sqrt{1-e^{-2 \mathcal{J}_{0}}}}{\mathcal{J}_{0} e^{-\mathcal{J}_{0}}} \approx 2.48$, may not be attained for $g \tau_{c} \approx \frac{1}{\sqrt{2 N_{\max }}}$. Then, a sudden change of $N$ should be undertaken as a function of $g \tau_{c}$ in order to optimize the estimation: For $g \tau_{c}$ lower than a certain critical value shown in Fig. 4, the best precision is achieved by the single-pulse Hahn echo $(N=1)$. However, if $g \tau_{c}$ is larger than this critical value, then the CPMG sequence with $N=N_{\max }$ is optimal. Qualitatively similar considerations apply for generalized Ornstein-Uhlenbeck processes. 
To sum up, the critical behavior of the environmental information revealed here is a fundamental feature that facilitates the attainment of the highest estimation precision of the environment memory-time. It represents an alternative characterization of the probe-qubit dynamics under suitable control or observation that leads to a phase transition on the dynamical behavior [44, 63-67]. Intriguingly, the absence of information has been shown to provide a distinctive signature of the environmental noise estimation.

Such information may be useful e.g., for studying molecular diffusion at the nanoscale and thereby characterizing biological systems [8, 39-41] or chemical-shift effects [7]; charge diffusion in conducting crystals [42] or spin diffusion in complex spin-networks [5, 43-45, 68]. Knowledge of the memory time may also be important for studying fundamental effects, such as quantum phase transitions in a spin environment $[46,47]$ or nonlocal correlations within a composite environment [48-51].

G.A.A. acknowledges the support of the European Commission under the Marie Curie Intra-European Fellowship for Career Development grant no. PIEF-GA-2012328605. G.K. acknowledges the ISF support under the Bikura (Prime) grant.

[1] A. Mittermaier and L. Kay, Science 312, 224 (2006).

[2] J. M. Taylor, P. Cappellaro, L. Childress, L. Jiang, D. Budker, P. R. Hemmer, A. Yacoby, R. Walsworth, and M. D. Lukin, Nat. Phys. 4, 810 (2008).

[3] G. Balasubramanian, I. Y. Chan, R. Kolesov, M. Al-Hmoud, J. Tisler, C. Shin, C. Kim, A. Wojcik, P. R. Hemmer, A. Krueger, T. Hanke, A. Leitenstorfer, R. Bratschitsch, F. Jelezko, and J. Wrachtrup, Nature 455, 648 (2008).

[4] I. Almog, Y. Sagi, G. Gordon, G. Bensky, G. Kurizki, and N. Davidson, J. Phys. B: At. Mol. Opt. Phys. 44, 154006 (2011).

[5] G. A. Álvarez and D. Suter, Phys. Rev. Lett. 107, 230501 (2011).

[6] J. Bylander, S. Gustavsson, F. Yan, F. Yoshihara, K. Harrabi, G. Fitch, D. G. Cory, Y. Nakamura, J. Tsai, and W. D. Oliver, Nat. Phys. 7, 565 (2011).

[7] P. E. S. Smith, G. Bensky, G. A. Álvarez, G. Kurizki, and L. Frydman, Proc. Natl. Acad. Sci. U. S. A. 109, 5958 (2012).

[8] G. A. Álvarez, N. Shemesh, and L. Frydman, Phys. Rev. Lett. 111, 080404 (2013).

[9] G. Kucsko, P. C. Maurer, N. Y. Yao, M. Kubo, H. J. Noh, P. K. Lo, H. Park, and M. D. Lukin, Nature 500, 54 (2013).

[10] P. Neumann, I. Jakobi, F. Dolde, C. Burk, R. Reuter, G. Waldherr, J. Honert, T. Wolf, A. Brunner, J. H. Shim, D. Suter, H. Sumiya, J. Isoya, and J. Wrachtrup, Nano Lett. 13, 2738 (2013).

[11] D. M. Toyli, C. F. d. 1. Casas, D. J. Christle, V. V. Dobrovitski, and D. D. Awschalom, Proc. Natl. Acad. Sci. U. S. A. 110, 8417 (2013).

[12] S. Steinert, F. Ziem, L. T. Hall, A. Zappe, M. Schweikert, N. Götz, A. Aird, G. Balasubramanian, L. Hollenberg, and
J. Wrachtrup, Nat. Commun. 4, 1607 (2013).

[13] M. S. Grinolds, M. Warner, K. D. Greve, Y. Dovzhenko, L. Thiel, R. L. Walsworth, S. Hong, P. Maletinsky, and A. Yacoby, Nat. Nanotechnol. 9, 279 (2014).

[14] A. Sushkov, I. Lovchinsky, N. Chisholm, R. Walsworth, H. Park, and M. Lukin, Phys. Rev. Lett. 113, 197601 (2014).

[15] A. Abragam, Principles of Nuclear Magnetism (Oxford University Press, London, 1961).

[16] C. P. Slichter, Principles of Magnetic Resonance (Springer Berlin Heidelberg, Berlin, Heidelberg, 1990).

[17] L. Viola and S. Lloyd, Phys. Rev. A 58, 2733 (1998).

[18] L. Viola, E. Knill, and S. Lloyd, Phys. Rev. Lett. 82, 2417 (1999).

[19] P. Zanardi, Phys. Lett. A 258, 77 (1999).

[20] K. Khodjasteh and D. Lidar, Phys. Rev. Lett. 95, 180501 (2005).

[21] G. S. Uhrig, Phys. Rev. Lett. 98, 100504 (2007).

[22] A. G. Kofman and G. Kurizki, Phys. Rev. Lett. 93, 130406 (2004).

[23] A. G. Kofman and G. Kurizki, IEEE Trans. Nanotechnology 4, 116 (2005).

[24] A. G. Kofman and G. Kurizki, Phys. Rev. Lett. 87, 270405 (2001).

[25] J. Clausen, G. Bensky, and G. Kurizki, Phys. Rev. Lett. 104, 040401 (2010).

[26] G. Gordon, N. Erez, and G. Kurizki, J. Phys. B: At. Mol. Opt. Phys. 40, S75 (2007).

[27] G. Gordon, G. Kurizki, and D. A. Lidar, Phys. Rev. Lett. 101, 010403 (2008).

[28] G. Kurizki and A. Zwick, in "From coherent to incoherent dynamical control of open quantum systems", To appear in Adv. Chem. Phys. 159, edited by P. Brumer, S. A. Rice, and A. R. Dinner (John Wiley, 2015).

[29] J. Clausen, G. Bensky, and G. Kurizki, Phys. Rev. A 85, 052105 (2012).

[30] G. Gordon and G. Kurizki, Phys. Rev. Lett. 97, 110503 (2006).

[31] G. Gordon and G. Kurizki, New J. Phys. 10, 045005 (2008).

[32] H. Uys, M. J. Biercuk, and J. J. Bollinger, Phys. Rev. Lett. 103, 040501 (2009).

[33] K. Khodjasteh, D. A. Lidar, and L. Viola, Phys. Rev. Lett. 104, 090501 (2010).

[34] A. Ajoy, G. A. Álvarez, and D. Suter, Phys. Rev. A 83, 032303 (2011).

[35] A. Zwick, G. A. Álvarez, G. Bensky, and G. Kurizki, New J. Phys. 16, 065021 (2014).

[36] B. M. Escher, G. Bensky, J. Clausen, and G. Kurizki, J. Phys. B: At. Mol. Opt. Phys. 44, 154015 (2011).

[37] D. Petrosyan, G. Bensky, G. Kurizki, I. Mazets, J. Majer, and J. Schmiedmayer, Phys. Rev. A 79, 040304 (2009).

[38] G. Bensky, D. Petrosyan, J. Majer, J. Schmiedmayer, and G. Kurizki, Phys. Rev. A 86, 012310 (2012).

[39] A. Zwick, G. A. Alvarez, and G. Kurizki, arXiv preprint arXiv:1507.03281 (2015).

[40] N. Shemesh, G. A. Álvarez, and L. Frydman, Journal of Magnetic Resonance 237, 49 (2013).

[41] N. Shemesh, G. A. Álvarez, and L. Frydman, PLoS ONE 10, e0133201 (2015).

[42] A. Feintuch, A. Grayevsky, N. Kaplan, and E. Dormann, Phys. Rev. Lett. 92, 156803 (2004). 
[43] D. Suter and R. R. Ernst, Phys. Rev. B 32, 5608 (1985).

[44] G. A. Álvarez, D. Suter, and R. Kaiser, Science 349, 846 (2015).

[45] G. A. Álvarez, R. Kaiser, and D. Suter, Annalen der Physik 525, 833 (2013).

[46] P. Haikka, J. Goold, S. McEndoo, F. Plastina, and S. Maniscalco, Phys. Rev. A 85, 060101 (2012).

[47] M. Gessner, M. Ramm, H. Haeffner, A. Buchleitner, and H.-P. Breuer, EPL (Europhysics Letters) 107, 40005 (2014).

[48] A. Smirne, S. Cialdi, G. Anelli, M. G. A. Paris, and B. Vacchini, Phys. Rev. A 88, 012108 (2013).

[49] E.-M. Laine, H.-P. Breuer, J. Piilo, C.-F. Li, and G.-C. Guo, Phys. Rev. Lett. 108, 210402 (2012).

[50] B.-H. Liu, D.-Y. Cao, Y.-F. Huang, C.-F. Li, G.-C. Guo, E.M. Laine, H.-P. Breuer, and J. Piilo, Scientific Reports 3 (2013), 10.1038/srep01781.

[51] S. Wissmann and H.-P. Breuer, Phys. Rev. A 90, 032117 (2014).

[52] A. Rivas, S. F. Huelga, and M. B. Plenio, Rep. Prog. Phys. 77, 094001 (2014).

[53] H.-P. Breuer, E.-M. Laine, J. Piilo, and B. Vacchini, arXiv:1505.01385 [quant-ph] (2015), arXiv: 1505.01385.

[54] S. Braunstein and C. Caves, Phys. Rev. Lett. 72, 3439 (1994).

[55] M. G. A. Paris, International Journal of Quantum Informa- tion 07, 125 (2009).

[56] B. M. Escher, R. L. de Matos Filho, and L. Davidovich, Nat. Phys. 7, 406 (2011).

[57] C. Benedetti and M. G. Paris, Physics Letters A 378, 2495 (2014).

[58] A. Kofman, G. Kurizki, and B. Sherman, Journal of Modern Optics 41, 353 (1994).

[59] D. Gelbwaser-Klimovsky, R. Alicki, and G. Kurizki, Phys. Rev. E 87, 012140 (2013).

[60] G. Kurizki, E. Shahmoon, and A. Zwick, Phys. Scr., in press Year of Ligth Special Issue (2015).

[61] H. Y. Carr and E. M. Purcell, Phys. Rev. 94, 630 (1954).

[62] S. Meiboom and D. Gill, Rev. Sci. Instrum. 29, 688 (1958).

[63] G. A. Álvarez, E. P. Danieli, P. R. Levstein, and H. M. Pastawski, J. Chem. Phys. 124, 194507 (2006).

[64] E. Danieli, G. Álvarez, P. Levstein, and H. Pastawski, Solid State Commun. 141, 422 (2007).

[65] I. Rotter, J. Phys. A: Math. Theor. 42, 153001 (2009).

[66] J. P. Garrahan and I. Lesanovsky, Phys. Rev. Lett. 104, 160601 (2010).

[67] I. Lesanovsky, M. van Horssen, M. Gutua, and J. P. Garrahan, Phys. Rev. Lett. 110, 150401 (2013).

[68] C. O. Bretschneider, G. A. Álvarez, G. Kurizki, and L. Frydman, Phys. Rev. Lett. 108, 140403 (2012). 